\title{
Finite-Size Scaling Study of the Surface and Bulk Critical Behavior in the Random-Bond 8-state Potts Model
}

\author{
Christophe Chatelain and Bertrand Berche: \\ Laboratoire de Physique des Matériaux, Université Henri Poincaré, Nancy 1, B.P. 239, \\ F-54506 Vandouvre les Nancy Cedex, France \\ (October 27, 1997, to appear in Phys. Rev. Lett.)
}

\begin{abstract}
The self-dual random-bond eight-state Potts model is studied numerically through large-scale Monte Carlo simulations using the Swendsen-Wang cluster flipping algorithm. We compute bulk and surface order parameters and susceptibilities and deduce the corresponding critical exponents at the random fixed point using standard finite-size scaling techniques. The scaling laws are suitably satisfied. We find that a belonging of the model to the 2D Ising model universality class can be conclusively ruled out, and the dimensions of the relevant bulk and surface scaling fields are found to take the values $y_{h}=1.849, y_{t}=0.977, y_{h_{s}}=0.54$, to be compared to their Ising values: $15 / 8,1$, and $1 / 2$.

PACS numbers: 64.60.Cn,64.60.Fr, 05.50.+q,05.70.Jk
\end{abstract}

The understanding of the role played by impurities on the nature of phase transitions is of great importance, both from experimental and theoretical perspectives. It is a quite active field of research where the resort to large-scale Monte Carlo simulations is often necessary [1]. The effect of quenched bond randomness in a system which undergoes, in the homogeneous case, a secondorder phase transition has been extensively studied. It is well understood since Harris proposed a relevance criterion for the fluctuating interactions [2]. Disorder appears to be a relevant perturbation when the specific heat exponent $\alpha$ of the pure system is positive. Since in the two-dimensional Ising model (IM) $\alpha$ vanishes due to the logarithmic Onsager singularity, this model was carefully studied in the '80s [3].

The analogous situation when the pure system exhibits a first-order transition was less well studied, in spite of the early work of Imry and Wortis who argued that quenched disorder could induce a secondorder phase transition [4]. This argument was then rigourously proved by Aizenman and Wehr, and Hui and Berker [5]. In two dimensions, even an infinitesimal amount of quenched impurities changes the transition into a second-order one. The first intensive Monte Carlo study of the effect of disorder at a first-order phase transition is due to Chen, Ferrenberg and Landau (CFL). These authors studied the $q=8$-state two-dimensional Potts model, which, in the pure case, is known to exhibit a first-order phase transition when $q>4[6]$. They definitively showed that the transition becomes secondorder in the presence of bond randomness [7], and obtained the critical exponents from a finite-size scaling (FSS) study at the critical point of a self-dual disordered system [8]. Their results, together with other related works 10,11, suggested that any two-dimensional random system should belong to the 2D pure IM universality class 12]. In a recent paper, Cardy and Jacobsen (CJ) used a different approach [13], based on a transfer matrix formalism [14], to study random-bond Potts models for different values of $q$. Their estimation of the critical exponents leads to a continuous variation of $\beta_{b} / \nu$ with $q$. This result is in accordance with previous theoretical calculations when $q \leq 4$ [15, and, in the randomnessinduced second-order phase transition regime $q>4$, it is quite different from the Ising value of $\frac{1}{8}$, and in sharp disagreement with the Monte Carlo results of Ref. [8] for $q=8$.

The surface properties of dilute magnetic systems paid less attention. Quite generally, the scaling laws involving surface and/or bulk exponents can be deduced from a homogeneity assumption for both surface and bulk singular free energies e.g.

$$
f_{\text {surf }}\left(t, h, h_{s}\right)=b^{-(d-1)} f_{\text {surf }}\left(b^{y_{t}} t, b^{y_{h}} h, b^{y_{h_{s}}} h_{s}\right) .
$$

All the standard critical exponents can be expressed in terms of the anomalous dimensions $y_{i}$ associated to the relevant scaling fields [16]. This makes their determination of great importance in the case of random systems. The $(1,1)$ surface of the Ising model on a square lattice has only recently been investigated through Monte Carlo simulations by Selke et al [17]. The critical exponent $\beta_{1}$ of the surface magnetization was found to be very close to its value in the pure 2D IM.

In this letter, we report a FSS study of the bulk and surface critical properties of the 8-state random-bond Potts model. Although this model has already been studied by Monte Carlo simulations, our approach is the first investigation of the surface properties for a random system other than Ising-like. It leads furthermore to different results in which concerns the bulk properties, and our aim is to bring some clear evidence to solve the discrepancy between the recent results of CJ (Ref. [13]) and those of CFL (Ref. [8]).

In the following, we consider the $q=8$-state randombond Potts model on the square lattice. The Hamiltonian of the system with quenched random interactions is written 


$$
-\beta \mathcal{H}=\sum_{(i, j)} K_{i j} \delta_{\sigma_{i}, \sigma_{j}}
$$

where the spins take the values $\sigma=1,2, \ldots, q$ and the sum goes over nearest-neighbor pairs $(i, j)$. The coupling strengths are allowed to take two different values $K_{1}=K$ and $K_{2}=K r$ with probabilities $p$ and $1-p$ respectively. The ratio $K_{2} / K_{1}$ is kept to the constant value $r=10$ (a strong enough value in order to ensure that the critical behavior is no longer governed by the pure system fixed point) for all the simulations. If both couplings occur with the same probability, $p=0.5$, the system is, on average, self-dual, and the critical point is exactly given by the critical line of the usual anisotropic model [1], 18]: $\left(\mathrm{e}^{K_{c}}-1\right)\left(\mathrm{e}^{K_{c} r}-1\right)=q$. At $p=0.5$, we performed large-scale simulations of $L \times L$ lattices $(10 \leq L \leq 96)$ with periodic boundary conditions in one direction (vertical direction) and free boundaries (FBC system) in the other direction. An equal number of couplings of both type is first distributed over all the bonds of the lattice. The couplings are then mixed randomly. This procedure ensures an exact probability $p=0.5$, and avoids the fluctuations around this value which would result from the use of a random number generator to build the distributions of couplings. The multi-spin coding and the Swendsen-Wang cluster flipping method 19] were used, and the histogram technique allowed us to determine the behavior of the different quantities over a range of $K$ [20]. For each distribution of the couplings, between $2 \times 10^{5}$ (smaller lattice sizes) to $4 \times 10^{5}$ (larger lattice sizes) Monte Carlo steps per spin were performed. Although it is smaller than the calculations of Ref. [8], this is always larger than $10^{4}$ times the correlation time, and turns out to be sufficient in order to produce reliable thermal averages. On the other hand, around 30 disorder realizations were performed in Ref. [8], but, since the averages over randomness are still strongly fluctuating (Fig. 1), we used $500(10 \leq L \leq 32), 330$ (40 $\leq L \leq 64)$, and $250(72 \leq L \leq 96)$ disorder realizations. (For smaller lattice sizes, the configurational fluctuations of data due to randomness are more pronounced, and a larger number of configurations is needed). These values guarantee the same order of magnitude for the contributions to the statistical errors resulting from the thermal average and from the replica average.

The translational invariance is restored in the vertical direction by averaging over the disorder realizations. The local order parameter for a given replica, written $\mu_{j}$, is defined by the majority orientation of the spins at column $j$ 21:

$$
\mu_{j}=\left(\frac{q \rho_{\max }(j)-1}{q-1}\right) .
$$

Here, $\rho_{\max }(j)=\max _{\sigma}\left(\rho_{\sigma}(j)\right)$, where $\rho_{\sigma}(j)$ is the density of spins in the state $\sigma$ at column $j$. The thermal average over the Monte Carlo iterations, written with brackets $\langle\ldots\rangle$, is performed and the physical quantities are then averaged over disorder configurations, for example $m_{j} \equiv\left[\left\langle\mu_{j}\right\rangle\right]$, where $[\ldots]$ denotes the replica average. The local surface susceptibility is given by $\chi_{11}=K L\left[\left\langle\mu_{1}^{2}\right\rangle-\left\langle\mu_{1}\right\rangle^{2}\right]$ and similar quantities are defined for the bulk.

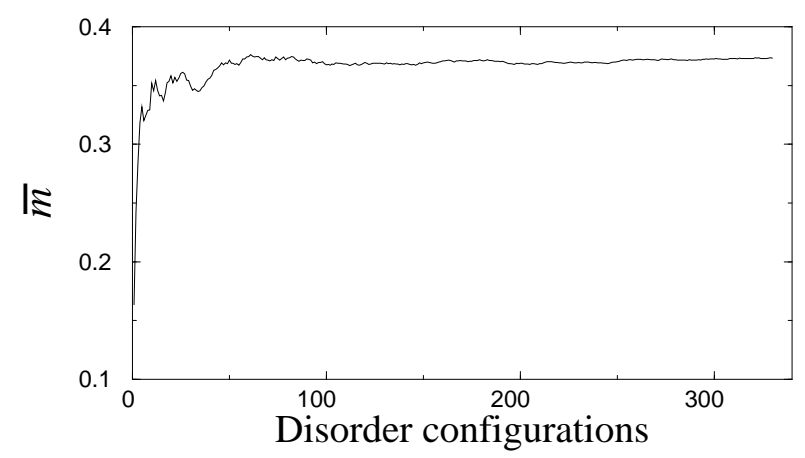

FIG. 1. Fluctuations of the averages over the number of realizations of disorder. Average total magnetization for $L=56$ over up to 330 replicas.

The first part of our analysis was to study the local magnetic surface properties. The local surface magnetization $m_{1}=\left[\left\langle\mu_{1}\right\rangle\right]$ is expected to follow the usual finite-size scaling behavior at the infinite lattice critical point: $m_{1}\left(K_{c}, L\right) \sim L^{-x_{1}}$, where the critical dimension $x_{1}=\beta_{1} / \nu$ is deduced from the size dependence of $m_{1}$, $\left[\left\langle\mu_{1}^{2}\right\rangle\right]$, and $\left[\left\langle\mu_{1}^{4}\right\rangle\right]$ (Fig. 2).

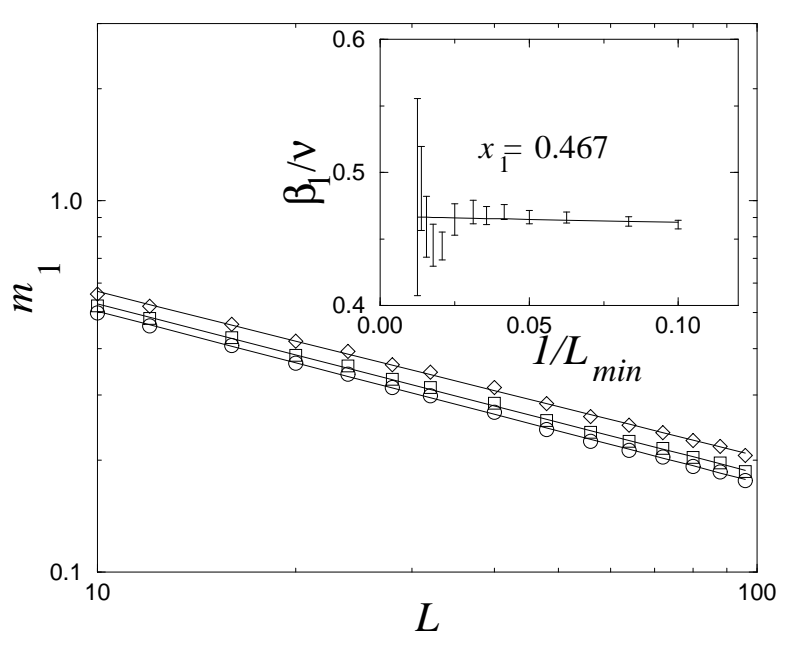

FIG. 2. Log-log plot of $\left[\left\langle\mu_{1}\right\rangle\right]$ (circles), $\left[\left\langle\mu_{1}^{2}\right\rangle\right]^{1 / 2}$ (squares), and $\left[\left\langle\mu_{1}^{4}\right\rangle\right]^{1 / 4}$ (diamonds) vs $L$. The slopes over the whole range of values of $L$ are respectively $-0.461,-0.456$, and -0.443 . The insert shows the effective surface exponent $x_{1}\left(L_{\min }\right)$ defined in the text vs $L_{\min }^{-1}$ (deduced from $\left.m_{1}\right)$ and its extrapolated value.

A power-law fit of the curve between a given smaller size $L_{\min }$ and the maximal value $L_{\max }=96$ defines an ef- 
fective exponent $x_{1}\left(L_{m i n}\right)$. The smaller size is then cancelled from the data and the whole procedure is repeated until the three larger sizes only remain. The effective exponent is plotted against $L_{\min }^{-1}$ (Insert in Fig. 2), and the critical exponent follows from the extrapolation at infinite size in the linear regime. Here the final estimation gives:

$$
\frac{\beta_{1}}{\nu}=0.467 \pm 0.006,
$$

where the uncertainty is the standard deviation.

The behavior of the local surface susceptibility $\chi_{11}$ is more ambiguous, since $\chi_{11}$ seems to exhibit a power-law behavior with a very small exponent, but also fits with a logarithmic divergence as it is the case for the pure IM. From the behaviors of $\chi_{11}\left(K_{c}\right)$ and $\chi_{11}^{\max }$ (deduced from histogram reweighting), we obtain $\gamma_{11} / \nu=0.099 \pm 0.009$ (Fig. 3). It corresponds to a fit which gives greater place to large sizes. On the other hand a logarithmic behavior seems also convincing (Fig. 3). Although this first analysis does not allow any definitive conclusion, the scaling relation $2 \beta_{1} / \nu+\gamma_{11} / \nu=d-1$ is best satisfied, within error bars, by the power-law case and is furthermore satisfying with the value of $x_{1}$ which rules out the $2 \mathrm{D}$ IM universality class.

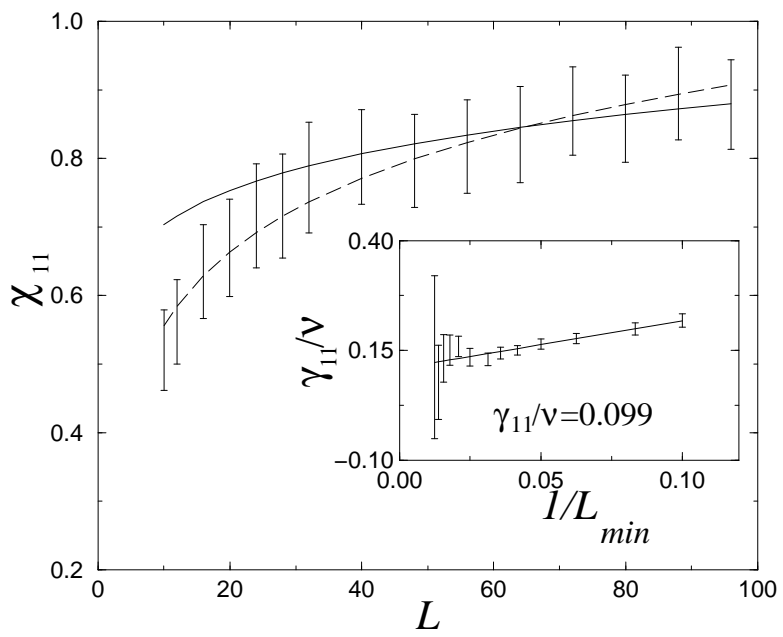

FIG. 3. Local susceptibility $\chi_{11}\left(K_{c}\right)$ at the critical point vs $L$ and power-law fit (solid line) or logarithmic fit (dashed line). The insert shows the effective exponent $\gamma_{11} / \nu$.

Once the local surface properties have been studied and since they strongly suggest that the model does not belong to the 2D IM universality class, a carefull analysis of bulk properties is needed to bring definitive conclusions. Bulk properties are furthermore needed in order to compute surface excess magnetization. For this purpose, we made simulations on $L \times L$ lattices with periodic boundary conditions in both directions (PBC system). The average quantities over the whole system lead to the critical exponents associated to the bulk magnetization and bulk susceptibility (Fig. 1). The determination of the slopes in the log-log plots, and of the corresponding standard deviations (of the order of $10 \%$ ), indicates large fluctuations. We then turned back to the "effective exponent" technique presented above to have accurate estimations. One thus obtains

$$
\frac{\beta_{b}}{\nu}=0.153 \pm 0.003, \quad \frac{\gamma_{b}}{\nu}=1.701 \pm 0.008 .
$$

The first value is very different from the IM value and from the result of Ref. [8] (0.126) and closer to the result of Ref. [13] (0.142). The second value satisfies to better than $0.7 \%$ the scaling law resulting from Rushbrooke and hyperscaling relations: $\gamma_{b} / \nu=d-2 \beta_{b} / \nu$. The correlation length exponent $\nu$ is deduced from the deviation of the effective critical coupling (at the maximum of the bulk susceptibility $\chi_{b}^{\max }$ ) from its exact value, $\left|K_{c}(L)-K_{c}\right| \sim L^{-1 / \nu}$ (Fig. 价. It leads to the correlation length exponent $\nu=1.023 \pm 0.020$ (Fig. 伍) [22].

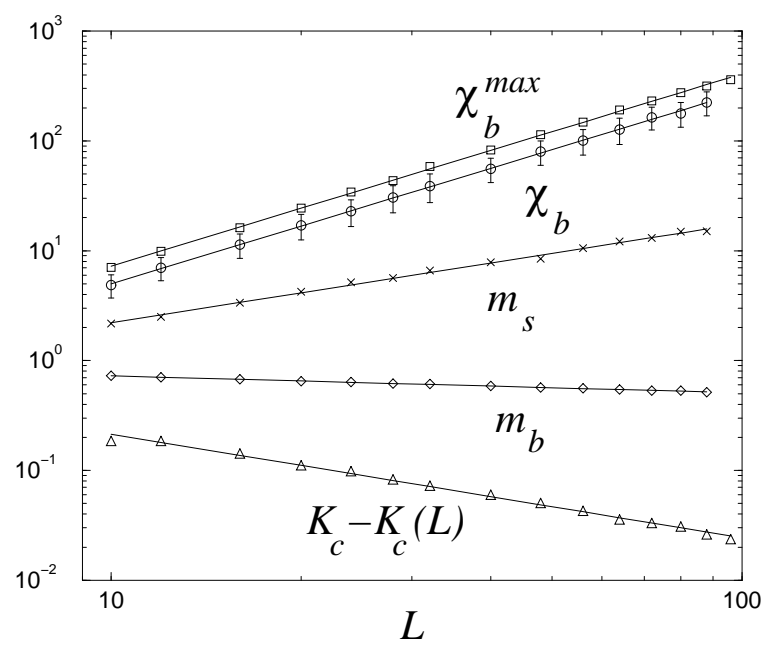

FIG. 4. Bulk quantities: Maximum of the susceptibility $\left(\chi_{b}^{\max }\right.$, squares $)$ and its value at $K_{c}\left(\chi_{b}\right.$, circles $)$; average magnetization $\left(m_{b}\right.$, diamonds); deviation of the effective critical coupling from its exact value (triangles), and surface excess magnetization $\left(m_{s}\right.$, crosses $)$. The corresponding exponents $\gamma_{b} / \nu,-\beta_{b} / \nu,-1 / \nu$, and $-\beta_{s} / \nu$ are given in the text.

Excess surface magnetization can be calculated by a comparison between the FBC and $\mathrm{PBC}$ systems:

$$
m_{s}=\frac{1}{2} \sum_{j=1}^{L}\left(m_{b}-m_{j}\right) \simeq \frac{1}{2} L\left(m_{b}-\bar{m}\right)
$$

where $\bar{m}$ is the average magnetization for the FBC system and $m_{b}$ for the PBC one. In Eq. 6, the approximation symbol renders the possible difference between the majority spin orientation on a layer $j$ and its value for the whole system. It produces a small difference between mean magnetization and mean profile for the FBC system but on average the two quantities should scale the same way. The corresponding exponent obtained from FSS takes the value: 


$$
\frac{\beta_{s}}{\nu}=-0.852 \pm 0.007
$$

It is in accordance with the expected value resulting from the scaling law $\beta_{s} / \nu=\beta_{b} / \nu-1=-0.847$, albeit $m_{s}$, given by a difference, is subject to strong fluctuations.

In this letter, we reported the results of large-scale Monte Carlo simulations [23] of the surface and bulk critical behaviors of a randomness-induced second-order phase transition in the 8-state Potts model. Concerning the bulk critical exponents, there is a clear discrepancy between our results and those of Ref. [8] which were very close to the pure IM values. The main difference between our procedure and these previous simulations is due to our use of a larger space of allowed coupling strengths. The self-dual condition $p=1 / 2$ was indeed imposed by these authors in both directions. A possible weakening of randomness could result of this choice. We furthermore generated a number of disorder realisations 10 times larger, which makes our results reliable. On the other hand, our value for $\beta_{b} / \nu$ is slightly above Cardy and Jacobsen's result, while we used the same order parameter than CFL. The possible explanation suggested in CJ (Ref. 13]) for the disagreement with CFL (non-standard order parameter) has thus to be dismissed.

We have also reported here the first extensive numerical study of surface critical behavior in a randomnessinduced second-order phase transition. While excess magnetization offers an alternate determination of the scaling dimension of the bulk magnetic field, local surface properties lead to the scaling dimension of a surface field which is also relevant.

We can summerize all the results in a table of the anomalous dimensions of the relevant scaling fields (Table (1). The independent determinations of these values, very close together, give reliability to the results. The final estimations are the following: $y_{t}=0.977, y_{h}=1.849$, $y_{h_{s}}=0.54$.

TABLE I. Scaling dimensions of the bulk and surface fields and of the temperature deduced from the values of the critical exponents.

\begin{tabular}{lcccccc}
\hline \hline & $\beta_{1} / \nu$ & $\gamma_{11} / \nu$ & $\nu$ & $\beta_{b} / \nu$ & $\gamma_{b} / \nu$ & $\beta_{s} / \nu$ \\
\hline$y_{h_{s}}$ & 0.533 & 0.549 & - & - & - & - \\
$y_{t}$ & - & - & 0.977 & - & - & - \\
$y_{h}$ & - & - & - & 1.847 & 1.850 & 1.852 \\
\hline
\end{tabular}

Finally, one has to mention that we also computed profiles and correlations (details will be published elsewhere). The values of $\eta=0.29$ (correlations PBC system), and of the critical exponents difference $x_{1}-x_{b}=$ 0.27 (profile close to the free surfaces, FBC system) lead to results which are slightly too small compared to the previous values of $x_{1}$ and $x_{b}$. Surprinsingly, the bulk exponent $x_{b}=0.145$ is found to be very close to Cardy and Jacobsen's result which was deduced from the behavior of correlations as well, but within a strip geometry.
We thank the Ciril and the Centre Charles Hermite in Nancy for computational facilities.

* To whom correspondence should be addressed, Electronic address: berche@lps.u-nancy.fr

$\dagger$ The Laboratoire de Physique des Matériaux is Unité Mixte de Recherche C.N.R.S. No 7556.

[1] W. Selke, L.N. Shchur and A.L. Talapov, In Annual Reviews of Computational Physics, Vol 1, edited by D. Stauffer (Singapore, World Scientific, 1994), p. 17.

[2] A.B. Harris, J. Phys. C 7, 1671 (1974).

[3] B.N. Shalaev, Phys. Rep. 237, 129 (1994).

[4] Y. Imry and M. Wortis, Phys. Rev. B 19, 3580 (1979).

[5] M. Aizenman and J. Wehr, Phys. Rev. Lett. 62, 2503 (1989), K. Hui and A.N. Berker, Phys. Rev. Lett. 62, 2507 (1989).

[6] F.Y. Wu, Rev. Mod. Phys. 54, 235 (1982).

[7] S. Chen, A.M. Ferrenberg, and D.P. Landau, Phys. Rev. Lett. 69, 1213 (1992).

[8] S. Chen, A.M. Ferrenberg, and D.P. Landau, Phys. Rev. E 52, 1377 (1995).

[9] S. Chen and D.P. Landau, Phys. Rev. E 55, 40 (1997).

[10] M.A. Novotny and D.P. Landau, Phys. Rev. B 24, 1468 (1981), M. Kardar, A.L. Stella, G. Sartoni, and B. Derrida, Phys. Rev. E 52, R1269 (1995).

[11] S. Wiseman and E. Domany, Phys. Rev. E 51, 3074 (1995).

[12] J.L. Cardy, J. Phys. A 29, 1897 (1996).

[13] J.L. Cardy and J.L. Jacobsen, Phys. Rev. Lett. 79, 4063 (1997).

[14] H.W.J. Blöte and M.P. Nightingale, Physica A 112, 405 (1982).

[15] Vl. Dotsenko, M. Picco and P. Pujol, Nucl. Phys. B455 [FS], 701 (1995).

[16] K. Binder, In Phase Transitions and Critical Phenomena Vol. 8, edited by C. Domb and J.L. Lebowitz (London, Academic Press, 1983), p. 1.

[17] W. Selke, F. Szalma, P. Lajkó, and F. Iglói, condmat/9707336 preprint.

[18] R. Fisch, J. Stat. Phys. 18, 111 (1978), W. Kinzel and E. Domany, Phys. Rev. B 23, 3421 (1981).

[19] R.H. Swendsen and J.S. Wang, Phys. Rev. Lett. 58, 86 (1987).

[20] A.M. Ferrenberg and R.H. Swendsen, Phys. Rev. Lett. 61, 2635 (1988), A.M. Ferrenberg and R.H. Swendsen, Phys. Rev. Lett. 63, 1195 (1989).

[21] M.S.S. Challa, D.P. Landau, and K. Binder, Phys. Rev. B 34, 1841 (1986).

[22] Other estimations of $\nu$ can in principle be obtained by the size behavior of $\left.\frac{d \ln X}{d K}\right|_{K_{c}}$ where $X$ is some quantity (magnetization for example), but they lead to estimations of $\nu$ slightly below 1 , while the stability of the random fixed point requires $\nu \geq 1$.

[23] The total CPU time used was around $9000 \mathrm{~h}$ on SGIPower Challenge Array RS-10000 processors. 